$05,13,01$

\title{
Влияние электрического поля на ферромагнитный резонанс в плоскослоистой магнитной системе
}

\author{
(C) E.A. Караштин ${ }^{1,2}$, A.A. Фраерман ${ }^{1,2}$ \\ ${ }^{1}$ Институт фоизики микроструктур РАН, \\ Нижний Новгород, Россия \\ ${ }^{2}$ Нижегородский государственный университет им. Н.И. Лобачевского, \\ Нижний Новгород, Россия \\ E-mail: eugenk@ipmras.ru
}

\begin{abstract}
Исследовано влияние электрического поля на ферромагнитный резонанс (ФМР) в многослойной магнитной системе, состоящей из двух магнитных слоев, разделенных тонкой немагнитной прослойкой. Показано, что собственные частоты ФМР при возбуждении колебаний намагниченности магнитным СВЧ-полем зависят от приложенного в плоскости слоев постоянного электрического поля. Также продемонстрировано, что в данной системе возможно возбуждение высокочастотных колебаний намагниченности электрическим СВЧ-полем. Результаты изучения поляризационных свойств механизма возбуждения показывают возможность экспериментального наблюдения рассмотренного эффекта.
\end{abstract}

Работа выполнена при поддержке гранта Российского научного фонда № 16-12-10340 (разделы 1, 2, 4, 5) и гранта РФФИ № 16-32-00527 (раздел 3).

\section{1. Введение}

Магнитоэлектрический эффект - взаимосвязь магнитного момента и электрической поляризации системы - активно исследуется в свете перспектив применения его в новых устройствах спинтроники [1-3]. Он наблюдается в средах, неинвариантных по отношению к обращению времени и пространственной инверсии [4]. Данный эффект был впервые обнаружен в $\mathrm{Cr}_{2} \mathrm{O}_{3}$ [5-7]. Позднее были открыты и подробно исследованы другие мультиферроики, такие как $\mathrm{BiFeO}_{3}[8], \mathrm{TbMnO}_{3}$, $\mathrm{DyMnO}_{3}, \mathrm{TbMn}_{2} \mathrm{O}_{5}$ [9-14]. В перечисленных соединениях нарушение симметрии по отношению к операции инверсии достигается за счет нецентросимметричности кристаллической структуры.

В том случае, если отсутствие центра инверсии в системе обусловлено пространственно неоднородным распределением намагниченности, данный эффект называют флексомагнитоэлектрическим. Впервые такой эффект был предсказан в работе [15]. Связь указанного явления со спиновыми токами вызвала значительный интерес к эффекту в недавнее время [16-19]. Проводились исследования как равновесного эффекта, так и эффекта, возникающего во внешнем магнитном поле [20]. Важно заметить, что флексомагнитоэлектрический эффект тесно связан со спиновым эффектом Холла [21].

Экспериментальных работ, посвященных флексомагнитоэлектрическому эффекту, крайне мало. В работах [22,23] показано, что доменная стенка в ферродиэлектрике смещается под действием электрического поля иглы, находящейся вблизи образца. Косвенным признаком флексоэлектрической природы данного эффекта является зависимость величины сдвига от магнитного поля для стенок смешанного типа (неелевская плюс блоховская), обладающих разной киральностью [24,25]. В $[26,27]$ численно продемонстрировано, что с помощью заряженной иглы можно перевести магнитную наночастицу в форме диска из вихревого состояния в антивихревое и обратно. Прямое наблюдение флексомагнитоэлектрического эффекта недавно проведено методом спектроскопии единичных молекул в висмутзамещенном феррите-гранате $\mathrm{Bi}: \mathrm{Lu}_{3} \mathrm{Fe}_{5} \mathrm{O}_{12}$, неоднородность намагниченности которого возникала за счет близости со сверхпроводником In, находящимся в смешанном состоянии во внешнем магнитном поле [28]. Экспериментов, посвященных измерению константы флексомагнитоэлектрического эффекта, в настоящее время не существует.

В настоящей работе теоретически исследовано обусловленное магнитоэлектрическим эффектом влияние электрического поля на ферромагнитный резонанс в плоскослоистой системе, состоящей из двух неколлинеарно намагниченных слоев, разделенных изолирующей прослойкой.

\section{2. Симметрийные соображения}

Влияние электрического поля на спиновые волны, вызванное флексомагнитоэлектрической связью, было теоретически рассчитано для среды с пространственно неоднородной намагниченностью $\mathbf{M}=\mathbf{M}(\mathbf{r})$ в [29]. В этой работе поправка к энергии системы, вызванная связью электрической поляризации и магнитного момента, записана феноменологически. Получим аналогичное феноменологическое выражение для плоскослоистой среды, состоящей из двух ферромагнитных слоев, разделенных диэлектрической прослойкой (рис. 1). Равновесный тензор спиновых токов $j_{i k}$ в такой системе отличен от нуля в случае, когда намагниченности слоев $\mathbf{M}_{1}$ и $\mathbf{M}_{2}$ неколлинеарны [30,31]. Он меняет знак при операции пространственной инверсии и сохраняет при обращении времени. Ограничиваясь вторым порядком по намагниченности, в обменном приближении $j_{i k}$ 


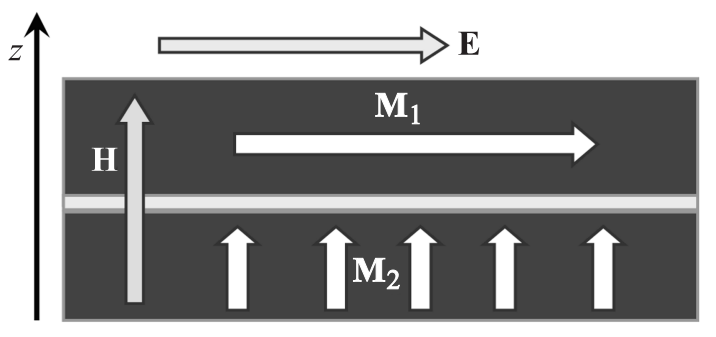

Рис. 1. Плоскослоистая магнитная система, постоянное магнитное поле Н приложено перпендикулярно слоям (вид сбоку).

можно записать в виде

$$
j_{i k}=\kappa n_{i}\left[\mathbf{M}_{1} \times \mathbf{M}_{2}\right]_{k},
$$

где $\mathbf{n}$ - единичный вектор нормали к слоям (направленный от первого слоя ко второму), $\kappa$ - константа. Электрическая поляризация среды Р имеет вид

$$
P_{l}=u e_{i k l} j_{i k}=\chi_{e}\left[\mathbf{n} \times\left[\mathbf{M}_{1} \times \mathbf{M}_{2}\right]\right]_{l},
$$

$e_{i k l}$ - тензор Леви-Чивита, $\chi_{e}-$ магнитоэлектрическая константа. Поскольку формула (2) не обладает обменной симметрией, она дает отличную от нуля поляризацию среды лишь при учете спин-орбитального взаимодействия. При наличии внешнего электрического поля $\mathbf{E}$ объемная плотность свободной энергии системы будет содержать вклад

$$
\delta F=\chi_{e}\left([\mathbf{n} \times \mathbf{E}],\left[\mathbf{M}_{1} \times \mathbf{M}_{2}\right]\right) .
$$

Введем обозначение $\mathbf{a}=[\mathbf{n} \times \mathbf{E}]$, тогда а определяется проекцией поля $\mathbf{E}$ на плоскость слоев. Будем поэтому считать, что $\mathbf{E}$ лежит в данной плоскости. Наличие вклада в свободную энергию вида (3) означает, во-первых, что постоянное электрическое поле, приложенное к системе, должно влиять на частоту ферромагнитного резонанса, а во-вторых, что высокочастотные колебания магнитного момента могут быть возбуждены электрическим полем СВЧ-волны.

\section{3. Оценка магнитоэлектрической константы}

Для того чтобы оценить магнитоэлектрическую константу $\chi_{e}$, которая входит в (2) и (3), вернемся к модели непрерывной среды с неоднородной намагниченностью $\mathbf{M}(\mathbf{r})$. Феноменологическая формула (2) в этом случае приобретает вид $[2,18]$

$$
\mathbf{P}=\eta(\mathbf{M} \operatorname{div} \mathbf{M}+[\mathbf{M} \times \operatorname{rot} \mathbf{M}]) .
$$

Рассмотрим ферромагнитный металл. Для описания движения электронов проводимости воспользуемся классической моделью с учетом спина, развитой в работе
Ааронова и Штерна [32]. Гамильтониан в этом случае имеет вид

$$
H=\frac{\mathbf{p}^{2}}{2 m}+\frac{J}{\hbar}(\mathbf{I}, \mathbf{M})
$$

где I - собственный магнитный момент частицы, $J$ обменная константа. Здесь и далее предполагается, что намагниченность М нормирована на единицу. Гамильтониан (5) дает следующие уравнения движения:

$$
\begin{aligned}
m \ddot{x}_{i} & =-J\left(\mathbf{I}, \partial \mathbf{M} / \partial x_{i}\right), \\
\mathbf{i} & =-J[\mathbf{I} \times \mathbf{M}] .
\end{aligned}
$$

Решая уравнение (7) в первом порядке по параметру адиабатичности $\beta=\hbar|\dot{\mathbf{M}}| / J$, получим:

$$
\mathbf{I}=l_{0}\left(\mathbf{M}-\frac{1}{J}\left[\mathbf{M} \times \frac{\partial \mathbf{M}}{\partial x_{i}}\right]\right),
$$

где $\mathbf{l}_{\mathbf{0}}= \pm \hbar / 2$. Из формулы (8) видно, что поправка к $\mathbf{l}$ первого порядка по параметру адиабатичности при вычислении среднего по импульсу спинового тока $j_{i k}=\dot{x}_{i} \mathbf{l}_{k}$ дает выражение, аналогичное (1), для непрерывной среды.

Будем учитывать спин-орбитальное взаимодействие, необходимое для возникновения магнитоэлектрического эффекта, в виде рассеяния на примесях. С этой целью воспользуемся формулой для тока с учетом аномальной скорости $[33,34]$

$$
\mathbf{j}=-e \int\left(\mathbf{v}+\frac{m \alpha}{\tau^{\sigma}}[\mathbf{v} \times \mathbf{s}]\right) f d \mathbf{v} /(2 \pi)^{3} .
$$

Здесь $\alpha$ - константа спин-орбитального взаимодействия, $\tau^{\sigma}$ - время релаксации импульса, $f-$ функция распределения Ферми, $s$ - средний магнитный момент электрона, который в классическом описании связан с $\mathbf{I}$ соотношением $\mathbf{I}=(\hbar / 2) \mathbf{s}$. Поправка к току, возникающая из-за наличия аномальной скорости, приводит к разделению зарядов, которые в свою очередь создают в среде электрическое поле. В равновесии аномальная поправка к току в точности компенсируется током проводимости, который возникает в связи с этим электрическим полем: $\mathbf{j}_{E}=\sigma \mathbf{E}$. Приравнивая эти две величины, получаем равновесное электрическое поле, индуцированное неоднородной намагниченностью,

$$
\begin{gathered}
\mathbf{E}=-4 \pi \eta(\mathbf{M} \operatorname{div} \mathbf{M}+[\mathbf{M} \times \operatorname{rot} \mathbf{M}]), \\
\eta=-\frac{\hbar / \tau^{\sigma}}{8 \pi e} \frac{\sigma^{H}}{\sigma},
\end{gathered}
$$

где $\sigma^{H} / \sigma=m \alpha / \tau^{\sigma}$ - отношение аномальной холловской проводимости к проводимости среды. Интересно, что, согласно (11), с увеличением частоты соударений электронов проводимости с примесями величина магнитоэлектрического эффекта возрастает. Электрическая поляризация $\mathbf{P}$ может быть определена исходя из граничных условий. Для системы, состоящей из слоев с бесконечными латеральными размерами, Р равна нулю, 
поскольку электрическое поле $\mathbf{E}$ лежит в плоскости слоев. Таким образом, поляризация возникает за счет границ. Оценить ее можно как $\mathbf{P}=-\mathbf{E} / 4 \pi$. Видно, что с учетом этой оценки формула (10) соответствует феноменологическому выражению (4).

Найдем теперь величину магнитоэлектрической константы, используя (11). Для переходных металлов $\sigma^{H} / \sigma \sim 0.01$, время релаксации импульса $\tau^{\sigma} \sim 10^{-15} \mathrm{~s}$. Это дает значение $\eta \sim-0.25 \mathrm{mV}$. Предполагая для случая многослойной системы характерный пространственный масштаб изменения намагниченности на границе ферромагнитных материалов порядка $5 \mathrm{~nm}$, получим константу в формуле (2) $\chi_{e} \sim-500 \mathrm{~V} / \mathrm{cm}$ (намагниченности слоев $\mathbf{M}_{1}$ и $\mathbf{M}_{2}$ считаются нормированными на единицу). Эта величина оказывается довольно большой за счет малого масштаба изменения намагниченности и соответственно небольшого объема, в котором локализовано возникающее электрическое поле.

\section{4. Влияние электрического поля на ферромагнитный резонанс (ФМР)}

4.1. Изменение частоты ФМР под действием постоянного электрического поля. Рассмотрим сначала случай, в котором к плоскослоистой системе, состоящей из двух магнитных слоев, разделенных немагнитной (диэлектрической) прослойкой, приложены постоянные поля $\mathbf{E}$ и $\mathbf{H}$. Для нахождения спектра колебаний используется уравнение Ландау-Лифшица

$$
\dot{\mathbf{M}}_{i}=-\gamma\left[\mathbf{M}_{i} \times \mathbf{H}_{i}^{\mathrm{eff}}\right],
$$

при этом $\mathbf{H}_{i}^{\text {eff }}=-\delta F / \delta \mathbf{M}_{i}$ содержит в соответствии c (3) вклад вида $\chi_{e}\left[\mathbf{M}_{1} \times\left[\mathbf{M}_{2} \times \mathbf{a}\right]\right]$ для первого и $-\chi_{e}\left[\mathbf{M}_{1} \times\left[\mathbf{M}_{2} \times \mathbf{a}\right]\right]$ для второго слоя. Линеаризуя систему уравнений (12) вблизи равновесного состояния в постоянном внешнем магнитном поле Н (считается, что поле достаточно велико для того, чтобы намагнитить систему до насыщения: $H / M_{s}>4 \pi$ ), получим резонансные частоты

$$
\begin{gathered}
\omega_{1}^{\perp}=\gamma\left(H-4 \pi M_{s}\right), \\
\omega_{2}^{\perp}=\gamma \sqrt{\left(H-4 \pi M_{s}\right)^{2}+2 V^{2}}
\end{gathered}
$$

для случая $\mathbf{H} \| \mathbf{n}$ (рис. 1) и

$$
\begin{array}{r}
\left(\omega_{1,2}^{\perp}\right)^{2}=\gamma\left(H\left(H+4 \pi M_{s}\right)+V^{2} \pm b\right), \\
b=V \sqrt{V^{2} \cos ^{4} \varphi+4 H\left(H+4 \pi M_{s}\right) \sin ^{2} \varphi}
\end{array}
$$

для $\mathbf{H} \perp \mathbf{n}$ (рис. 2). Здесь $V=\chi_{e} E / M_{s}, \varphi-$ угол между $\mathbf{E}$ и $\mathbf{H}$; предполагается, что намагниченность насыщения для обоих слоев одинакова и равна $M_{s}$ (символы $\perp$ и $\|$ в обозначении частоты соответствуют случаям, когда Н перпендикулярно плоскости слоев и лежит в их плоскости соответственно). Как видно из (5), при $\mathbf{H} \| \mathbf{n}$ резонансная частота для одной из мод не зависит от электрического поля Е. Кроме того, в случае $\chi_{e} E / M_{s}^{2} \ll 1$ поправка, линейная по $\chi_{e} E$, остается только для $\mathbf{H} \perp \mathbf{n}$ и имеет максимум $\delta \omega_{1,2}= \pm \gamma V$ при $\mathbf{E} \perp \mathbf{H}$.

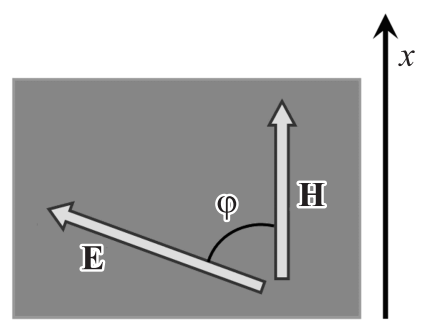

Рис. 2. Плоскослоистая магнитная система, постоянное магнитное поле $H$ лежит в плоскости слоев (вид сверху).

Данная конфигурация оптимальна для наблюдения сдвига резонансных частот электрическим полем.

Используя оценку константы $\chi_{e}$ и взяв $M_{s} \sim 1000 \mathrm{G}$ и поле $H=4 \pi M_{s}$, получим относительную величину сдвига резонансной частоты $\delta \omega / \omega \sim 6 \cdot 10^{-10}$ на $1 \mathrm{~V} / \mathrm{cm}$. Увеличение сдвига на один-два порядка может быть достигнуто путем подбора материала, в частности, с меньшим $M_{s}$. Однако очевидно, что для экспериментального наблюдения такого эффекта необходимо большое внешнее электрическое поле, что делает невозможным обнаружение его в системе, состоящей лишь из проводящих слоев. Представляется наиболее реальным наблюдение эффекта в структуре, в которой ферромагнетики разделены диэлектрической прослойкой, методом локального измерения ФМР [35] вблизи края пленки при приложении в его окрестности большого электрического поля.

4.2. Возбуждение колебаний намагниченности электрическим СВЧ-полем. В рамках решения задачи о возбуждении колебаний намагниченности электрическим полем волны вычислена флексоэлектрическая восприимчивость $\beta_{i j}^{1}, \beta_{i f}^{2}$ связывающая высокочастотную поправку к намагниченности $\mathbf{m}_{1,2} \mathbf{c}$ электрическим СВЧ-полем е $\left(m_{1,2 i}=\beta_{i j}^{1,2} e_{j}\right)$. Рассмотрим сначала случай $\mathbf{H} \perp \mathbf{n}$. Введем обозначение

$$
D_{\|}=\omega\left(\omega^{2}-\gamma^{2} H\left(H+4 \pi M_{s}\right)\right) \text {. }
$$

Линеаризуя, как и выше, (12), получаем следующие ненулевые компоненты $\beta_{i j}^{1}$ :

$$
\beta_{y x}^{\| 1}=\frac{i \omega^{2} \gamma \chi_{e} M_{s}^{2}}{D_{\|}}, \quad \beta_{z x}^{\| 1}=\frac{\omega \gamma^{2} \chi_{e} M_{s}^{2} H}{D_{\|}} .
$$

Декартова система координат введена таким образом, что $\mathbf{n}\|o z, \mathbf{H}\| o x$ (рис. 2). Вводя для случая $\mathbf{H} \| \mathbf{n}$ обозначение

$$
D_{\perp}=\omega\left(\omega^{2}-\gamma^{2}\left(H-4 \pi M_{s}\right)^{2}\right)
$$

и проведя аналогичные вычисления, получим

$$
\begin{gathered}
\beta_{x x}^{\perp 1}=-\beta_{y y}^{\perp 1}=-\omega \gamma^{2} \chi_{e} M_{s}^{2}\left(H-4 \pi M_{s}\right) / D_{\perp}, \\
\beta_{x y}^{\perp 1}=-\beta_{y x}^{\perp 1}=-i \omega^{2} \gamma \chi_{e} M_{s}^{2} / D_{\perp} .
\end{gathered}
$$

При этом в обоих случаях $\beta_{i j}^{2}=-\beta_{i j}^{1}$, что нетрудно понять, учитывая, что поправка к свободной энергии вида (3) антисимметрична по отношению к перестановке первого и второго слоев местами. 
Анализируя формулы (15)-(18), заметим, что при приложении Н в плоскости слоев колебания намагниченности возбуждаются компонентой е, параллельной $\mathbf{H}$, а при $\mathbf{H} \| \mathbf{n}$ - любой компонентой $\mathbf{e}$ в плоскости слоев. Для экспериментального наблюдения эффекта удобно выбрать $\mathbf{H} \| \mathbf{n}$ и поляризацию СВЧ-волны, в которой магнитное поле $\mathbf{h} \| \mathbf{n}$ (не возбуждает колебаний намагниченности), а электрическое $\mathbf{e} \perp \mathbf{n}$.

Важно отметить, что в этом случае в отличие от рассмотренного в предыдущем подразделе, экспериментальное наблюдение эффекта общепринятыми методами измерения спектра ФМР представляется реальным. Вопервых, это связано с тем, что электрическое СВЧ-поле хорошо проникает в металлические структуры толщиной порядка нескольких десятков нанометров. Вовторых, размещение образца в области СВЧ-резонатора, где магнитное СВЧ-поле близко к нулю, и ориентация его по отношению к электрическому и магнитному полям СВЧ-излучения, как указано выше, позволяют практически избежать генерации высокочастотных колебаний магнитным полем СВЧ-излучения, нежелательной в данном эксперименте.

\section{5. Заключение}

В работе теоретически показано, что собственные частоты ферромагнитного резонанса системы, состоящей из двух магнитных слоев, разделенных тонкой немагнитной прослойкой, зависят от приложенного в плоскости слоев постоянного электрического поля. Максимальный сдвиг частоты реализуется в случае, когда внешние постоянные магнитное и электрическое поля лежат в плоскости слоев и при этом взаимно перпендикулярны. Оценки показали, что данный эффект крайне мал, поэтому представляется реальным его обнаружение с использованием методов локального магнитного отклика (таких как магнитно-резонансная силовая микроскопия) вблизи источника сильного электрического поля.

Также продемонстрировано, что в указанной системе возможно возбуждение высокочастотных колебаний намагниченности переменным электрическим СВЧ-полем. При приложении постоянного магнитного поля в плоскости слоев для возбуждения колебаний намагниченности электрическим полем СВЧ-волны она должна быть поляризована таким образом, чтобы вектор переменного электрического поля был сонаправлен с постоянным магнитным полем. В том случае, если постоянное магнитное поле перпендикулярно плоскости слоев, роль в возбуждении колебаний намагниченности играет проекция электрического поля СВЧ-волны на плоскость слоев. Последняя конфигурация является оптимальной для экспериментального наблюдения эффекта, поскольку позволяет выбрать поляризацию СВЧ-излучения таким образом, чтобы переменное магнитное поле было сонаправлено с постоянным и не играло роли в возбуждении высокочастотных колебаний намагниченности. Экспериментальное обнаружение рассмотренного явления позволит определить константу флексомагнитоэлектрического эффекта в такой системе.

\section{Список литературы}

[1] M. Fiebig, N.A. Spaldin. Eur. Phys. J B 71, 293 (2009).

[2] А.П. Пятаков, А.К. Звездин. УФН 182, 593 (2012).

[3] А.П. Пятаков, А.С. Сергеев, Е.П. Николаева, Т.Б. Косых, А.В. Николаев, К.А. Звездин, А.К. Звездин. УФН 185, 1077 (2015).

[4] Л.Д. Ландау, Е.М. Лифшиц. Электродинамика сплошных сред. Наука, М. (1982). 624 с.

[5] И.Е. Дзялошинский. ЖЭТФ 37, 881 (1959).

[6] Д.Н. Астров. ЖЭТФ 38, 984 (1960).

[7] V.J. Folen, G.T. Rado, E.W. Stalder. Phys. Rev. Lett. 6, 607 (1961).

[8] А.К. Звездин, А.П. Пятаков. УФН 174, 465 (2004).

[9] T. Kimura, S. Ishihara, H. Shintani, T. Arima, K.T. Takahashi, K. Ishizaka, Y. Tokura. Phys. Rev. B 68, 060403(R) (2003).

[10] T. Kimura, T. Goto, H. Shintani, K. Ishizaka, T. Arima, Y. Tokura. Nature (London) 426, 55 (2003).

[11] T. Goto, T. Kimura, G. Lawes, A.P. Ramirez, Y. Tokura. Phys. Rev. Lett. 92, 257201 (2004).

[12] T. Kimura, G. Lawes, T. Goto, Y. Tokura, A.P. Ramirez. Phys. Rev. B 71, 224425 (2005).

[13] D. Higashiyama, S. Miyasaka, N. Kida, T. Arima, Y. Tokura. Phys. Rev. B 70, 174405 (2004).

[14] N. Hur, S. Park, P.A. Sharma, J.S. Ahn, S. Guha, S-W. Cheong. Nature (London) 429, 392 (2004).

[15] В.Г. Барьяхтар, В.А. Львов, Д.А. Яблонский. Письма в ЖЭТФ 37, 565 (1983).

[16] Q-F. Sun, H. Guo, J. Wang. Phys. Rev. B 69, 054409 (2004).

[17] H. Katsura, N. Nagaosa, A.V. Balatsky. Phys. Rev. Lett. 95, 057205 (2005)

[18] P. Bruno, V.K. Dugaev. Phys. Rev. B 72, 241302(R) (2005).

[19] M. Mostovoy. Phys. Rev. Lett. 96, 067601 (2006).

[20] K.T. Delaney, M. Mostovoy, N.A. Spaldin. Phys. Rev. Lett. 102, 157203 (2009).

[21] J. Sinova, S.O. Valenzuela, J. Wunderlich, C.H. Back, T. Jungwirth. Rev. Mod. Phys. 87, 1213 (2015).

[22] А.С. Логгинов, Г.А. Мешков, А.В. Николаев, А.П. Пятаков. Письма в ЖЭТФ 86, 124 (2007).

[23] A.S. Logginov, G.A. Meshkov, A.V. Nikolaev, E.P. Nikolaeva, A.P. Pyatakov, A.K. Zvezdin. Appl. Phys. Lett. 93, 182510 (2008).

[24] А.С. Сергеев, Д.А. Сечин, О.В. Павленко, Е.П. Николаева, А.В. Николаев, Т.Б. Косых, А.П. Пятаков. Изв. РАН. Сер. физ. 77, 1523 (2013).

[25] A.P. Pyatakov, D.A. Sechin, A.S. Sergeev, A.V. Nikolaev, E.P. Nikolaeva, A.S. Logginov, A.K. Zvezdin. Europhys. Lett. 93, 17001 (2011).

[26] A.P. Pyatakov, G.A. Meshkov, A.K. Zvezdin. J. Magn. Magn. Mater. 324, 3551 (2012).

[27] A.P. Pyatakov, A.S. Sergeev, F.A. Mikailzase, A.K. Zvezdin. J. Magn. Magn. Mater. 383, 255 (2015).

[28] I.S. Veshchunov, S.V. Mironov, W. Magrini, V.S. Stolyarov, A.N. Rossolenko, V.A. Skidanov, J.-B. Trebbia, A.I. Buzdin, Ph. Tamarat, B. Lounis. Phys. Rev. Lett. 115, 027601 (2015).

[29] D.L. Mills, I.E. Dzyaloshinskii. Phys. Rev. B 78, 184422 (2008).

[30] J. Wang, K.S. Chen. J. Phys.: Condens. Matter 19, 236215 (2007).

[31] Y.G. Shen, Z.H. Yang. Europhys. Lett. 78, 17003 (2007).

[32] Y. Aharonov, A. Stern. Phys. Rev. Lett. 693593 (1992).

[33] S.K. Lyo, T. Holstein. Phys. Rev. Lett. 29, 423 (1972).

[34] S. Zhang. Phys. Rev. Lett. 85, 393 (2000).

[35] O. Klein, G. de Loubens, V.V. Naletov, F. Boust, T. Guillet, H. Hurdequint, A. Leksikov, A.N. Slavin, V.S. Tiberkevich, N. Vukadinovic. Phys. Rev. B 78, 144410 (2008). 